\title{
Comparación de los niveles de agresión entre hombres que ejercen violencia en la pareja y un grupo control, medidos con la versión chilena de la Escala de Agresión de Buss and Perry
} Comparison of aggression levels between a perpetrators of intimate partner violence group and a control group, assessed by the chilean version of the Buss and Perry Aggression Scale

Maruzzella Valdivia-Peralta ${ }^{1}$, Tatiana Sanhueza-Morales ${ }^{2}$, Luis González-Bravo ${ }^{3}$ y Fernando Quiroga-Dubornais ${ }^{4}$

\begin{abstract}
Background: Intimate partner violence is a serious public health problem, with a prevalence reaches $49 \%$ for psychological violence and 13\% physical violence. Aim: To compare the levels of aggression between a sample of men referred for domestic violence therapy $(n=34)$ and a nonclinical sample $(N=40)$, assessed by the Chilean version of the AQ scale. Method: Comparative analytical observational cross-sectional study. Results: Statistically significant differences in favor of the group of perpetrators was found, in overall AQ scores $(p=0.013)$, physical aggression subscale ( $p=0.005)$, anger subscale $(p=0.005)$ and hostility subscale $(p=0.000)$. No statistically significant differences were found in verbal aggression subscale $(p=0.705)$, despite being the clinical group scores higher than the control group. Conclusions: Results are partially consistent with findings from previous research. Results are discussed from an empirical and sociological perspective.
\end{abstract}

Key words: Aggression, Spouse abuse, Males.

Rev Chil Neuro-Psiquiat 2016; 54 (2): 133-140

Recibido: 30/11/2015

Aceptado: 29/02/2016

${ }^{*}$ Artículo desarrollado en el marco del Proyecto de Investigación DIUC, Universidad de Concepción, N²09.171.010.0.

Psicóloga Clínica, Magíster en Psicología de la Salud, Departamento de Psicología, Universidad de Concepción.

Asistente Social, Magíster en estudios de Género y Cultura mención Ciencias Sociales. Doctora $\odot$ en Servicio Social, Universidad Laval, Québec, Canadá. Escuela Trabajo Social, Universidad de Concepción. Concepción, Chile.

3 Psicólogo Clínico, Magíster en Investigación Social y Desarrollo, Escuela de Psicología y Vicerrectoría de Aseguramiento de la Calidad, Universidad San Sebastián. Concepción, Chile.

4 Kinesiólogo. Magíster en Dirección General de Empresas, Facultad de Ciencias de la Salud, Universidad San Sebastián, Concepción, Chile. 


\section{Introducción}

$\mathrm{L}$ a violencia en la pareja (IPV, Intimate Partner Violence, violencia por parte de la pareja íntima), es un grave problema de salud pública. Según los Centros para la Prevención y Control de Enfermedades, en Estados Unidos, provocó 2.340 muertes en el 2007, siendo de ellas 70\% mujeres y $30 \%$ hombres. Esto se traduce en costos de salud y pérdida de productividad, que llegan a más de 8 mil millones de dólares hoy día ${ }^{1}$.

En España ${ }^{2}$, en un estudio con 421 mujeres seleccionadas con un muestreo estratificado, se informa que el 20,2\% ha sufrido IPV y que el 6,2\% de ellas lo ha experimentado durante el último año.

En Chile, el primer estudio de prevalencia realizado en 1994 encontró que en una muestra de 1.000 mujeres de la Región Metropolitana, un $25,9 \%$ había vivido violencia física y un 33,9\% violencia psicológica. En tanto, en la ciudad de Temuco en el año 2001, en una muestra de 422 mujeres, se ha encontrado que un $49 \%$ reportan agresión psicológica, un $13 \%$ violencia física y un $5,5 \%$, violencia sexual ${ }^{3}$. En el año 2006 hubo 35 fallecimientos de mujeres por dicha problemática, y al 29 de septiembre del año 2007 ya habían 48, pese a la vigencia de la ley $20.066^{4}$.

En términos de modelos explicativos del fenómeno, se oscila desde aquellos derivados de la sociología y el feminismo, entendiendo el fenómeno asociado a las relaciones de poder y dominio que los hombres han sometido tradicionalmente a la mujer, hasta aquellos que lo entienden como hecho delictivo o un fenómeno biológico ${ }^{5,6}$. Uno de los modelos más ampliamente utilizado es el basado en los conceptos ecológicos de Brofenbrenner ${ }^{7}$, quien visualiza al ambiente en su conjunto como un grupo de estructuras seriadas y estructuradas, en donde cada nivel contiene al otro. Así en el Microsistema, el individuo participa directamente; en el Mesosistema, los miembros de diferentes microsistemas interactúan entre sí independiente de la persona central; el exosistema está constituido por las entidades y organizaciones a las que puede acceder el individuo o su familia; el macrosistema contiene la política, costumbres y en general aque- llos aspectos que representan el tejido cultural de la sociedad ${ }^{8}$.

Una tipología clásica de hombres que ejercen violencia en la pareja, y ampliamente citada en la literatura, es la elaborada por Holtzworth-Munroe \& Stuart (1994) quienes a partir de las dimensiones de severidad y generalidad de la violencia, y desórdenes de personalidad o psicopatología ${ }^{9}$ establecen: violencia ejercida sólo en la familia (en donde el abuso sería menor), violencia general/antisocial y disfórica/bordeline (en estos dos últimos perfiles, el abuso ejercido sería severo). En esta línea se encuentra la categorización de Loinaz, Echeburua y Torrubia (2010) que distingue agresores "violentos con la pareja/estables emocionalmente/integrados socialmente" y "violentos generalizados/poco estables emocionalmente/no integrados socialmente" ${ }^{10}$. En Chile ${ }^{11}$, también se ha publicado una clasificación que incluye dos tipologías psicológicas: el grupo de hombres que maltratan sólo en la familia y los más cercanos a la violencia antisocial. De cualquier forma, existe consenso en que la presencia de agresividad en los varones, constituye un factor de riesgo para ejercer IPV ${ }^{12}$.

Según Helfritz et $\mathrm{al}^{14}$, existen múltiples medidas en habla inglesa para evaluar el comportamiento agresivo en varones que ejercen IPV: el LHAQ - Lifetime History of Aggression Questionnaire, Cuestionario de Historia de Vida de Agresión- ${ }^{14}$, el STAXI -State- Trait Anger Expression Inventory, Inventario de Expresión de la Ira Estado Rasgo- ${ }^{15}$, incluso el MMPI -Minnesota Multiphasic Personality Inventory, Inventario Multifásico de Personalidad de Minnesota $-{ }^{16}$. Sin embargo, uno de los más usados es el Inventario de Agresión AQ de Buss and Perry o AQ (1992) (1, utilizado en la presente investigación), y que contiene cuatro subescalas que miden agresión física, agresión verbal, ira y hostilidad.

Ya en el año 2005, Norlander y Eckhardt ${ }^{17}$ en un interesante meta-análisis, indicaban que los perpetradores sistemáticamente informaban mayores niveles de enojo y hostilidad que los hombres no violentos a través de diferentes métodos de evaluación, entre ellos el cuestionario de Buss \& Dur$\mathrm{kee}^{18}$, o su versión abreviada el BAAQ -Brief AngerAggression Questionnaire, Cuestionario Breve de Ira 
Agresión- ${ }^{19}$, ambos precursores del AQ utilizado en la presente investigación. Helfritz et a ${ }^{13}$ así mismo informan que en un grupo de bajo riesgo de IPV, encontraron significativamente menores puntajes en el AQ que un grupo con antecedentes de IPV en las subescalas de ira $(\mathrm{p}<0,01)$ y hostilidad $(\mathrm{p}<0,01)$; además de diferencias estadísticamente significativas en el puntaje total AQ, con el grupo de alto riego de IPV $(p>0,05)$.

En la misma línea, Lee, Walters, Hall \& Basile ${ }^{20}$, después de estudiar a 340 hombres perpetradores, reportan que las diferencias significativas que hallan en los puntajes de la subescala de ira del AQ, se deberían a presencia de historia de violencia en la familia de origen $(t=-3,91 ; p<0,0001)$.

Smith \& Waterman ${ }^{21}$ mediante una batería de instrumentos, entre ellos el AQ, encontraron que tanto los hombres violentos como las mujeres violentas, eran más agresivos que estudiantes y delincuentes no violentos. Así mismo, eran igualmente propensos a reportar su agresión física e independiente del género, mostraban niveles similares de agresión y la impulsividad.

Finalmente, argumentos a favor de una relación de todas las escalas del AQ con actos de agresión directa e indirecta dirigido a las parejas y otras personas, se encuentran en los hallazgos de Archer \& Webb en el año 2006 22 . En particular indican que tres subescalas (física, verbal y hostilidad) están más fuertemente relacionadas con otras personas que con la pareja.

\section{Material y Método}

\section{Hipótesis de investigación}

Los hombres derivados a atención psicoterapéutica por IPV, presentarán significativamente mayores niveles de agresión que los hombres que se atienden por patologías generales de salud.

\section{Diseño}

Estudio observacional comparativo analítico de corte transversal.

\section{Muestra}

Las muestras Clínica y Control, siendo similares en términos sociodemográficos, se diferenciaron fundamentalmente en el motivo de atención en un servicio de salud: en el caso del primero, haber sido derivados a terapia psicológica por violencia intrafamiliar. En el grupo control, en tanto, problemas de salud general en consulta espontánea.

\section{Muestra clínica/experimental}

La muestra Clínico/Experimental no aleatoria estuvo constituida por 34 hombres, derivados a terapia psicológica por IPV, a centros de salud de la provincia del Bío-Bío en Chile. La edad promedio fue de 44,4 años y se atendieron en el Programa de Salud Mental del Hospital Higueras de Talcahuano $(58,82 \%)$ y en el Programa de Intervención con hombres que ejercen violencia contra su pareja mujer (41,18\%), de Gendarmería de Chile.

\section{Muestra control}

La muestra control estuvo constituida por 40 hombres, atendidos en el marco del convenio entre la Facultad de Ciencias de la Salud de la Universidad San Sebastián, y diferentes centros de salud de la provincia de Concepción para las carreras de Nutrición y Dietética, Kinesiología, Fonoaudiología y Terapia Ocupacional. La edad promedio de la muestra fue de 41,1 años. Los criterios de inclusión de esta segunda muestra fueron ser hombre, tener entre 21 y 75 años y estar en una relación de pareja. No se solicitó no tener antecedentes de violencia en la pareja, con el propósito de no sesgar la respuesta al cuestionario.

\section{Instrumentos}

\section{Cuestionario de Agresión AQ (Aggression}

Questionaire, Buss \& Perry 1992)

El instrumento original ${ }^{23}$ para medir agresividad, consta de 29 ítems de respuesta tipo Likert de cinco grados de 1 a 5 , que arroja puntajes entre 29 y 145 puntos. Además, contiene cuatro subescalas que miden agresión física, agresión verbal, ira y hostilidad.

La Escala ha sido validada en diferentes países, entre los cuales se puede mencionar España con dos estudios con muestras de 15 a 25 años de edad y 16 años a 84 , respectivamente $e^{24,25}$. 
En Colombia, se ha reportado una estructura de cinco factores ${ }^{26}$, mientras que en Argentina ${ }^{27}$ se informan dos factores en un estudio factorial exploratorio, que más tarde son redefinidos en una estructura de 2 y 4 factores en el análisis confirmatorio.

En la validación realizada en Chile y utilizada en esta investigación ${ }^{28}$ se obtuvo una confiabilidad medida con alfa de Cronbach de $\alpha=0,89$, y una estructura de cuatro factores. Se reporta además adecuada validez de criterio con otras escalas de agresión e impulsividad.

\section{Aspectos éticos}

Al momento de la aplicación, a las personas participantes en el estudio se les leyó y explicó consentimiento informado aprobado para el proyecto de investigación $\mathrm{N}^{\circ}$ 209.171.010.0. En el caso de estar de acuerdo y mediante él, los sujetos accedieron a participar y a entregar información fidedigna, y por parte de las(os) investigadores, a mantener su anonimato y a hacer uso de la información exclusivamente para cumplir los objetivos del estudio.

\section{Resultados}

En la Tabla 1, se muestran los resultados descriptivos de las variables estudiadas, es decir, para cada subescala de la escala AQ y el puntaje total, en cada uno de los grupos (Clínico y Control).

Tal como se puede observar en la Tabla 1, los puntajes obtenidos tanto en la escala total de agresión AQ como en cada una de las subescalas son superiores en el grupo Clínico/experimental -hombres derivados a terapia por violencia conyugal- a los puntajes del grupo control: Agresión Total (Grupo Clínico: 71,53 vs Grupo Control: 61,88); Agresión Física (Grupo Clínico: 19,88 vs Grupo Control: 15,04); Agresión Verbal (Grupo Clínico: 11,76 vs Grupo Control: 10,76); Ira (Grupo Clínico: 17,91 vs Grupo Control: 13,36) y Hostilidad (Grupo Clínico: 21,97 vs Grupo Control: 15,84).

Para verificar si las diferencias entre las puntuaciones obtenidas en las diferentes mediciones son estadísticamente significativas, se realizó la prueba de U de Mann-Whitney para las variables independientes. La siguiente tabla muestra los resultados.

Tal como se observa en la Tabla 2, en la columna de significancia, sólo existen diferencias estadísticamente significativas (a favor del grupo clínico en los puntajes de la escala AQ total $(\mathrm{p}=0,013)$, en la subescala de agresión física $(\mathrm{p}=0,005)$, en la subescala de ira $(p=0,005)$, y en la subescala de hostilidad ( $p=0,000)$. No se encontraron sin embargo, diferencias estadísticamente significativas en la subescala agresión verbal $(\mathrm{p}=0,705)$, a pesar de ser los puntajes del grupo experimental superiores al grupo control.

Tabla 1. Descriptivos para la escala total AQ y para las subescalas en ambos grupos

\begin{tabular}{|c|c|c|c|c|}
\hline & Media & $\begin{array}{c}\text { Desviación } \\
\text { Estándar }\end{array}$ & Mínimo & Máximo \\
\hline \multicolumn{5}{|l|}{ Grupo clínico/experimental } \\
\hline Subescala agresión física & 19,88 & 1,175 & 9 & 44 \\
\hline Subescala agresión verbal & 11,76 & 0,600 & 5 & 22 \\
\hline Subescala ira & 17,91 & 1,052 & 7 & 31 \\
\hline Subescala hostilidad & 21,97 & 0,980 & 14 & 33 \\
\hline Escala total & 71,53 & 3,041 & 41 & 116 \\
\hline \multicolumn{5}{|l|}{ Grupo control } \\
\hline Subescala agresión física & 15,04 & 1,128 & 9 & 36 \\
\hline Subescala agresión verbal & 10,76 & 0,762 & 5 & 21 \\
\hline Subescala ira & 13,36 & 0,897 & 8 & 28 \\
\hline Subescala hostilidad & 15,84 & 1,120 & 11 & 31 \\
\hline Escala total & 61,88 & 2,064 & 38 & 97 \\
\hline
\end{tabular}


Tabla 2. Resultados de prueba U de Mann Whitney para la escala AQ total y cada una de las subescalas

\begin{tabular}{|c|c|c|c|c|c|}
\hline & Muestras & $\mathbf{n}$ & Rangos & U de Mann-Whitney & Significancia \\
\hline Subescala agresión física & $\begin{array}{l}\text { Grupo experimental } \\
\text { Grupo control }\end{array}$ & $\begin{array}{l}34 \\
40\end{array}$ & $\begin{array}{l}48,40 \\
33,66\end{array}$ & 479,5 & $0,005^{\star}$ \\
\hline Subescala agresión verbal & $\begin{array}{l}\text { Grupo experimental } \\
\text { Grupo control }\end{array}$ & $\begin{array}{l}34 \\
40\end{array}$ & $\begin{array}{l}41,12 \\
39,16\end{array}$ & 727,000 & 0,705 \\
\hline Subescala ira & $\begin{array}{l}\text { Grupo experimental } \\
\text { Grupo control }\end{array}$ & $\begin{array}{l}34 \\
40\end{array}$ & $\begin{array}{l}48,31 \\
33,72\end{array}$ & 482,500 & $0,005^{\star}$ \\
\hline Subescala hostilidad & $\begin{array}{l}\text { Grupo experimental } \\
\text { Grupo control }\end{array}$ & $\begin{array}{l}34 \\
40\end{array}$ & $\begin{array}{l}50,72 \\
31,90\end{array}$ & 400,500 & $0,000^{\star}$ \\
\hline Escala total & $\begin{array}{l}\text { Grupo experimental } \\
\text { Grupo control }\end{array}$ & $\begin{array}{l}34 \\
40\end{array}$ & $\begin{array}{l}44,24 \\
31,78\end{array}$ & 451,000 & $0,013^{\star *}$ \\
\hline
\end{tabular}

${ }^{\star} \mathrm{p}<0,01 ;{ }^{* *} \mathrm{p}<0,05$.

\section{Discusión}

Este es el primer estudio realizado en Chile que, utilizando una versión de un instrumento para medir agresión validado en el país, explore una problemática de salud pública significativa.

Los hallazgos confirman la hipótesis de investigación, en la medida que se encontraron significativamente mayores niveles de agresión medidos a través de la escala AQ en el grupo de hombres que ejercen IPV. Más específicamente, en lo concerniente a las subescalas de ira y hostilidad, concuerdan en gran medida con los resultados reportados por Norlander y Eckhardt ${ }^{17}$ quienes en su revisión de 33 estudios con 28 muestras independientes encuentran mayores niveles de ira y hostilidad en los perpetradores que en hombres no violentos. En ese sentido no es sorprendente encontrar mayores niveles de agresión en hombres derivados a terapia por IPV.

También concuerda con los hallazgos de Helfritz et $\mathrm{al}^{13}$ quienes informan mayores puntajes en subescalas de ira, hostilidad y agresión total en hombres con alto riesgo de IPV; sin embargo, concuerdan sólo parcialmente con los hallazgos de Smith \& Waterman ${ }^{21}$ quienes indican que esta superioridad de los puntajes en la escala AQ se daría en todos los puntajes para los delincuentes violentos: agresión física, agresión verbal, ira y hostilidad $(\mathrm{p}<0,0005)$. De hecho, Archer $\& \mathrm{Webb}^{22}$ también encuentran que estas diferencias en agresión se presentan en la subescala de agresión verbal.

El hecho de que en agresión verbal pudieran no encontrarse diferencias entre la muestra clínica y el grupo control, tiene algunas explicaciones posibles desde la literatura. Son precisamente estas semejanzas con la población no clínica de varones, las que indican que la masculinidad es en sí misma un factor de riesgo para la violencia íntima ${ }^{6}$. Dicho de otra forma, la construcción cultural de la masculinidad legitima (independiente del grupo en estudio) el uso de la violencia en el lenguaje $e^{29-31}$.

Más que una naturalización del uso de la violencia verbal, esto debe ser visto como una alerta y factor de riego, ya que existen antecedentes importantes de que la violencia verbal sería un precursor de la violencia física en la relación ${ }^{32,33}$.

En una mirada opuesta, sin embargo, es interesante atender lo señalado por Stockdale y $\mathrm{cols}^{32}$, quienes señalan que la agresión verbal no está necesariamente asociada a la física por diferentes razones. Habría una heterogeneidad en las vías a través de las cuales la primera desemboca en la segunda. Además, el uso de la agresión verbal es altamente dependiente del contexto, ya que parejas con alto estrés usan más la agresión verbal, siendo más un estado en parejas románticas que un rasgo. Por último, indican que incluso algunas formas de violencia verbal, pueden ser una experiencia 
normativa menos dañina que la agresión física en las parejas.

Así, la violencia verbal sería mucho más generalizada en la población e incluso, las mujeres podrían llegar incluso a ser más agresivas verbalmente que los varones en los conflictos ${ }^{35,36}$.

En cualquier caso, sin embargo, los autores del siguiente artículo consideran que los principales aportes de la presente investigación son en primer lugar, la evidencia de diferencias en los niveles de agresión totales, en hostilidad, ira y agresión física con mayores puntajes hallados en hombres derivados a terapia psicológica. Segundo, la evidencia de ausencia de diferencias significativas en violencia verbal. Esto apunta a un uso más generalizado de ella en la población general y un factor de riesgo ecológico en la línea de lo señalado por AndrésPueyo et $\mathrm{al}^{7,37}$ : validación del uso de la violencia a nivel de creencias y valores sociales. Por último, en tercer lugar, la aplicación concreta de la versión chilena de la escala AQ a una problemática clínica real.

En el ámbito de la atención en salud, identificar la agresión total, en forma de hostilidad, agresión física e ira, como un factor de riesgo para otras patologías presentes en hombres que ejercen IPV y al mismo tiempo, atender la violencia verbal como un factor presente en las relaciones en la población general masculina, son un elemento de mucha utilidad para la atención en salud de los varones y sus respectivas parejas ${ }^{38}$.

Finalmente, dentro de las limitaciones de la presente investigación se pueden mencionar el tamaño muestral limitado, la selección no aleatoria de los participantes y el potencial sesgo en las respuestas de los hombres derivados a terapia.

\begin{abstract}
Resumen
Antecedentes: La violencia hacia la pareja es un grave problema de salud pública, con una prevalencia que llega al 49\% para la violencia psicológica y $13 \%$ para la violencia física. Objetivo: Comparar los niveles de agresión entre una muestra de hombres derivados a terapia por violencia intrafamiliar $(n=34)$ y una muestra no clínica $(n=40)$ mediante la versión chilena de la escala AQ. Método: Estudio observacional comparativo analítico de corte transversal. Resultados: Se encuentran diferencias estadísticamente significativas a favor del grupo de perpetradores en puntajes de AQ total ( $p=0,013)$, en la subescala de agresión física $(p=0,005)$, en la subescala de ira $(p=0,005)$ y en la subescala de hostilidad $(p=0,000)$. No se encontraron diferencias estadísticamente significativas en la subescala agresión verbal $(p=0,705)$, a pesar de ser los puntajes del grupo clínico superiores al grupo control. Conclusiones: Resultados concuerdan parcialmente con hallazgos de investigaciones anteriores. Se discuten resultados desde una perspectiva empírica y sociológica.
\end{abstract}

Palabras clave: Agresión, Violencia en la pareja, Hombres.

\section{Referencias bibliográficas}

1. CDC, Centers for Disease Control and Prevention. Understanding Intimate Partner Violence 2012. Disponible en http://www.cdc.gov/violenceprevention/pdf/ipv_factsheet-a.pdf [Consultado el 13 de noviembre de 2015].
2. Fontanil Y, Ezama E, Fernández R, Gil P, Herrero F, Paz D. Prevalencia del maltrato de pareja contra las mujeres. Psicothema 2005; 17: 90-5.

3. Vizcarra MB, Cortés J, Bustos L, Alarcón M \& Muñoz S. Violencia conyugal en la ciudad de Temuco: Un estudio de prevalencia y factores asociados. Rev Med Chile 2001; 129: 1405-12. 
4. Donoso E. Violencia contra la mujer en Chile: problema de salud pública. Rev Chil Obstet Ginecol 2007; 72 (5): 281-2.

5. Shah SS, Ayub Q, Firasat S, Kaiser F, Mehdi SQ. Y haplogroups and aggressive behavior in a Pakistani ethnic group. Aggress Behav 2009; 35: 68-74.

6. Bertsch K, Grothe M, Prehn K, Vohs K, Berger C, Hauenstein $\mathrm{K}$, et al. Brain volumes differ between diagnostic groups of violent criminal offenders. Eur Arch Psychiatry Clin Neurosci 2013; 263 (7): 593-606.

7. Andrés-Pueyo A, López S, Alvarez, E. Valoración del riesgo de violencia contra la pareja por medio de la SARA. Papeles de Psicólogo 2008; 29: 107-22.

8. Boon HJ, Cottrell A, King D, Stevenson RB, Millar J. Bronfenbrenner's bioecological theory for modelling community resilience to natural disasters. Nat Hazards 2012; 60 (2): 381-408.

9. Johnson R, Gilchrist E, Beech A, Weston S, Takriti R, Freeman R. Psychometric Typology of U.K. Domestic Violence Offenders. J Interpers Violence 2006; 21: 1270-85.

10. Loinaz I, Echeburua E, Torrubia R. Tipología de agresores contra la pareja en prisión. Psicothema 2010; 22: 106-11.

11. Barría J, Macchiavello A. Anatomía de los hombres que ejercen violencia hacia sus parejas: Primer levantamiento de datos para el diseño de un perfil del hombre maltratador. Revista Psicologia.com 2012; 16. Disponible en : http://www.psiquiatria. com/revistas/index.php/psicologiacom/article/ view/1470/

12. Capaldi DM, Knoble NB, Shortt JW, Kim HK. A systematic review of risk factors for intimate partner violence. Partner Abuse 2012; 3: 231-80.

13. Helfritz LE, Stanford MS, Conklin SM, Greve KW, Villemarette-Pittman N, Houston R. Usefulness of self-report instruments in assessing men accused of domestic violence. Psychol Rec 2006; 56: 17180.

14. Coccaro E, Berman M, Kavoussi R. Assessment of life history of aggression: development and psychometric characteristics. Psychiatry Research 1997; 73 (3): 147-57.

15. Spielberger C, Gorusch R, Luschene R. Manual for the state-trait anxiety inventory. Palo Alto:
Consulting Psychologists Press; 1970.

16. Butcher JN, Dahlstrom WG, Graham JR, et al. The Minnesota Multiphasic Personality Inventory-2 (MMPI-2) Manual for Administration and Scoring. Minneapolis: University of Minneapolis Press; 1989.

17. Norlander B, Eckhardt C. Anger, hostility, and male perpetrators of intimate partner violence: $A$ meta-analytic review. Clin Psychol Rev 2005; 25 : 119-52.

18. Buss AH, Durkee A. An inventory for assessing different kinds of hostility. J Consult Psychol 1957; 21 (4): 343-9.

19. Maiuro R, Vitaliano P, Cahn T. A Brief Measure for the Assessment of Anger and Aggression. J Interpers Violence June 1987; 2; 166-78

20. Lee RD, Walters ML, Hall JE, Basile KC. Behavioral and attitudinal factors differentiating male intimate partner violence perpetrators with and without a history of childhood family violence. J Fam Violence 2013; 28: 85-94.

21. Smith P, Waterman M. Self-Reported Aggression and Impulsivity in Forensic and Non-Forensic Populations: The Role of Gender and Experience. J Fam Violence 2006; 21: 425-37.

22. Archer J, Webb, I. The Relation Between Scores on the Buss-Perry Aggression Questionnaire and Aggressive Acts, Impulsiveness, Competitiveness, Dominance, and Sexual Jealousy. Aggress Behav 2006; 32: 464-73.

23. Buss AH, Perry M. The aggression questionnaire. J Pers Soc Psychol 1992; 63: 452-9.

24. Andreu JM, Peña ME, Graña JL. Adaptación psicométrica de la versión española del Cuestionario de Agresión. Psicothema 2002; 14, 476-82.

25. Vigil-Colet A, Lorenzo-Seva U, Codorniu-Raga M, Morales F. Factor structure of the Buss-Perry aggression questionnaire in different samples and languages. Aggressive Behavior. November 2005; 31 (6): 601-8.

26. Chahín-Pinzón N, Lorenzo-Seva U, Vigil-Colet A. Características psicométricas de la adaptación colombiana del Cuestionario de Agresividad de Buss y Perry en una muestra de preadolescentes y adolescentes de Bucaramanga. Universitas Psychologica 2012; 11 (3): 979-88. 
27. Reyna C, Lello Ivacevich M, Sánchez A, Brussino S. The Buss-Perry Aggression Questionnaire: Construct validity and gender invariance among Argentinean adolescents. International Journal of Psychological Research 2011; 4 (2): 30-7.

28. Valdivia M, Fonseca E, González L, Lemos S. Psychometric properties of the AQ Aggression Scale in a sample of Chilean students. Psicothema 2014; 26: 39-46.

29. Stoltz J. Masculinity and school violence: Addressing the role of male gender socialization. Canadian Journal of Counselling 2005; 39 (1): 52-63.

30. Cockerham W. Sociología de la Medicina. Madrid: Pearson; 2002.

31. Lewis L. Man Talk, Masculinity, and a Changing Social Environment. Caribbean Review of Gender Studies 2007; 1: 1-20.

32. Chung G, Tucker B, Li G, Zhou X, Hwang S. Exploratory analysis of verbal aggression in romantic relationships among unmarried men and women: Predictive patterns by gender and race. J Soc Pers Relat 2011; 28: 1005-23.
33. McHugh M, Rakowski S, Swiderski C. Men's Experience of Psychological Abuse: Conceptualization and Measurement Issue. Sex Roles 2013; 69: 16881.

34. Stockdale L, Tackett S, Coyne S. Sex differences in verbal aggression use in romantic relationships: a meta-analytic study and review. J Aggress Confl Peace Res 2013; 5: 167-78.

35. Winstok Z, Enoch G. Distribution of Verbal and Physical Violence for Same and Opposite Genders Among Adolescents. Am J Mens Health 2008; 2: 272-80.

36. Women 'are just as abusive as men'. The Herald 2014; 5 .

37. Stith $\mathrm{S}$, et al. Intimate partner physical abuse perpetration and victimization risk factors: a metaanalytic review 2004. Aggress Violent Behav; 10: 65-98.

38. Ruiz-Pérez I, Plazaola-Castano J, Del Río-Lozano M. Physical health consequences of intimate partner violence in Spanish women. Eur J Public Health 2007; 17: 437-43.
Correspondencia:

Luis González-Bravo

Escuela de Psicología y Vicerrectoría

de Aseguramiento de la Calidad, Universidad San Sebastián, Concepción, Chile.

E-mail: luis.gonzalezb@uss.cl 